\title{
UNILATERAL SPONTANEOUS TUBAL TWIN ECTOPIC PREGNANCY: A RARE OCCURRENCE
}

\author{
Pulei AN, Muga PA, Ongeti KW, Kinuthia J, Ogutu 0 \\ Correspondence to Dr. Anne Pulei, Department of Human Anatomy, University of Nairobi, PO Box 30197- \\ 00100, Nairobi, Kenya. Tel: +254722465924 E-mail: anmunkush@yahoo.com.
}

\begin{abstract}
Unilateral tubal twin pregnancy remains rare despite a rise in the incidence of singleton ectopic pregnancies. A 27-year-old Gravida 1 Para $0+0$ at 12 weeks gestation, presented to our institution with a 1-month history of lower abdominal pain, that progressively worsened and became very severe. An abdominal ultrasound revealed an extrauterine gestational sac that looked like a single viable fetus in the right adnexa at about 12 weeks by crown rump length. Free fluid was noted in the right iliac fossa and Morrison's pouch. A conclusion of a right-ruptured ectopic pregnancy was made. The patient underwent laparotomy and a diagnosis of twin right-sided fimbrial ectopic pregnancy was made. The crown rump lengths of the twins were $6 \mathrm{~cm}$ and $4 \mathrm{~cm}$. We present this case because unilateral tubal twin pregnancy is still a rare phenomenon, and clinicians as well as clinical embryologists need to acknowledge its existence considering the diagnosis of this case was not made pre-operatively.

Key words: Twin Tubal, ectopic pregnancy, unilateral
\end{abstract}

\section{INTRODUCTION}

Ectopic pregnancy (EP) is one of the leading causes of pregnancy related deaths in the first trimester. It is defined as a pregnancy in which the implantation of the conceptus occurs elsewhere other than the endometrial cavity. The commonest site of occurrence of EP is the Fallopian tubes (97\%), specifically, ampulla (55\%), isthmus (25\%), and fimbria (17\%), and in $3 \%$ of patients EP occurs in the abdominal cavity, ovary, or cervix (Lozeau and Potter 2005). Factors that increase the risk of EP include pelvic inflammatory disease, operative trauma, congenital anomalies, assisted reproductive therapy and adhesions. EP is an important cause of maternal morbidity and mortality especially in developing countries, where the majority of patients present late with rupture and hemodynamic compromise (Panti et al., 2012). Unilateral tubal twin pregnancy (UTTP) is a rare manifestation and was first reported by De Ott (1891), and since then more than 100 cases have been described as separate reports. In Kenya, the first report of UTTP was in 1990 by Lema. Its incidence is 1 in every 125,000 pregnancies and 1 in 200 ectopic pregnancies (Parker et al., 1999). Most cases are monozygotic monochorionic. This paper describes another case of UTTP, which was encountered at laparotomy.

\section{CASE REPORT}

A $27 y$ r old para $0+0 \mathrm{G} 1$ at 12 weeks and 6 days gestation, presented to our institution with a month long history of lower abdominal pain. The pain was of insidious onset, gradually worsened and became very severe. This pain was not relieved by any medication. She did not have vaginal discharge or bleeding at the time. She had no history of being treated for sexually transmitted diseases and had not had prior pelvic or tubal surgery. She had never smoked cigarettes. The patient reported no headache, dizziness, nausea or vomiting on the day of admission. She had not perceived quickening. Her menarche was at 16 years. Her menses had

Submitted $10^{\text {th }}$ October 2016, corrected $20^{\text {th }}$ October 2016. Published online $1^{\text {st }}$ December 2016 . To cite: Pulei AN, Muga PA, Ongeti KW, Ogutu O, Kinuthia J. 2017. Unilateral spontaneous tubal twin ectopic pregnancy: a rare occurrence. Anatomy Journal of Africa. 6: $820-823$. 
been regular, 28-day cycle that lasted 5 days and of normal flow. She had no history of using hormonal contraception or intrauterine devices. She reported that she is married and had primary school education level. She worked as a hairdresser, and lived with her husband. On the day of presentation, she was in a fair general condition and her vital signs were within normal. She had a widespread non- pruritic nonerythematous papular rash on her abdomen, which she had developed after applying a certain "herbal" medication for her pain. Generalized tenderness was noted on abdominal palpation. A pelvic mass was elicited and was about $20 \mathrm{~cm}$ from the pubic symphysis; it was mobile, nontender and firm. A pelvic examination revealed normal external genitalia, a closed external cervical os and right-sided adnexal tenderness as well as a brown non-foul smelling discharge on the examining finger.
Investigations carried out included a hemogram, which showed a low level of hemoglobin of $8.75 \mathrm{~g} / \mathrm{dL}$, hematocrit of $27.1 \%$, White blood cell count of $18.2 * 10^{9}$ and neutrophilia of $15.3 * 10^{9}$ $(83.8 \%)$. Her renal function tests were within normal with a urea of $6.7 \mathrm{mmol} / \mathrm{L}, \mathrm{Na} 132$ $\mathrm{mmol} / \mathrm{L}, \quad \mathrm{K} 4.1 \mathrm{mmol} / \mathrm{L}$ and creatine of 60.4 umol/L. A HIV test done on her was negative. She had a trans-abdominal ultrasound done which revealed a non-gravid uterus with multiple intramural myomas the largest measured 3.8 by $3.1 \mathrm{~cm}$. The endometrial lining was found to be about $4.4 \mathrm{~cm}$ thick. An extrauterine gestational sac with a single viable fetus in right adnexa at about 12 weeks by CRL was seen. Free fluid was noted in the right iliac fossa and Morrison's pouch. A conclusion of a right slow leaking ectopic was made and the patient underwent an exploratory laparotomy.
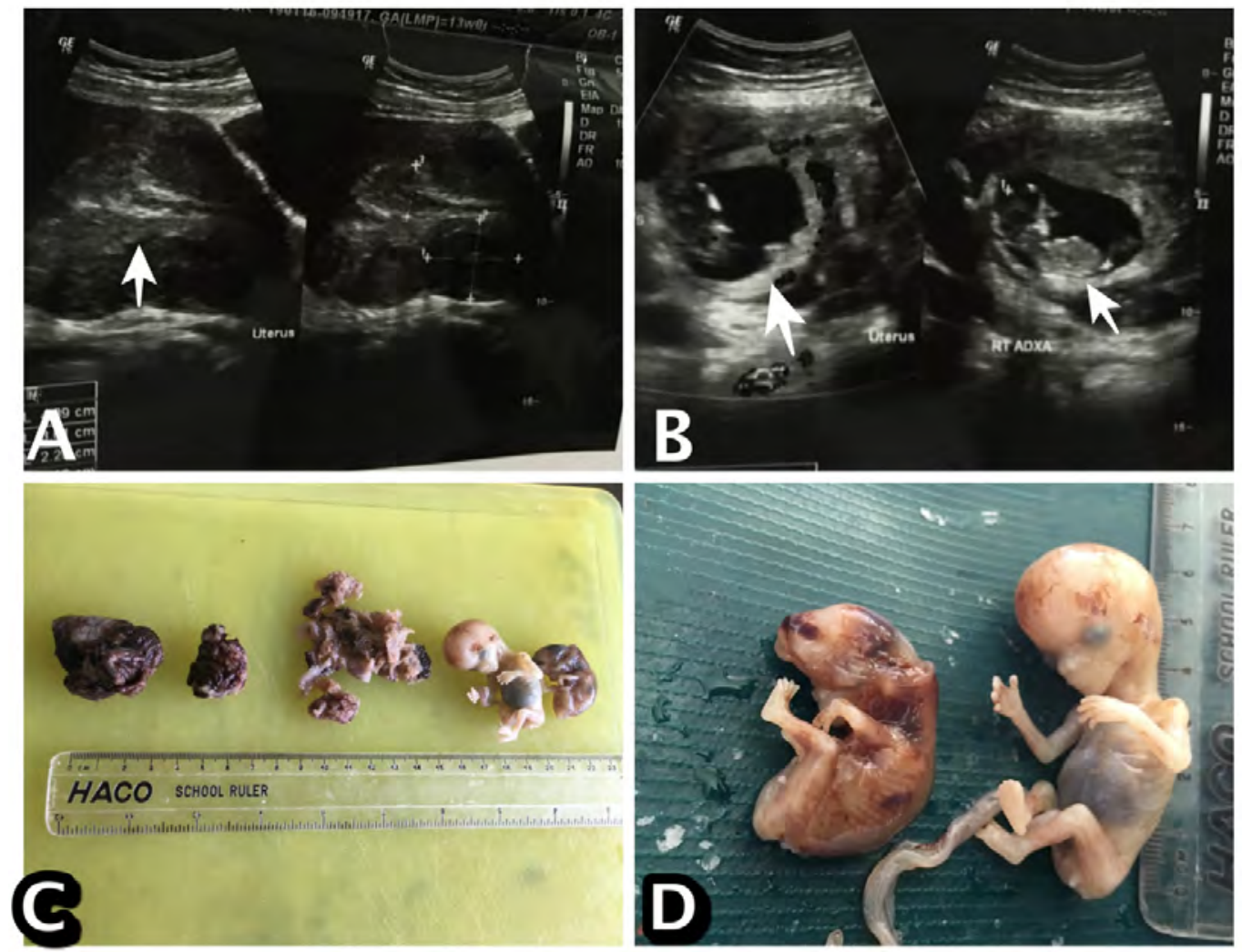

Fig. 1: Illustration of the twin ectopic. A shows an empty uterus (white arrows) and B shows a gestational sac in the right adnexa that was visualized as 1 fetal pole (white arrows) in this view through trans-abdominal ultrasound. C and $D$ are the findings at pathology. $C$ includes part of the tube: the ampulla as well as some placental tissue. D shows the CRL of the twins, 4 and 6 respectively. 
Intra-operatively, intra-peritoneal clots amounting to $300 \mathrm{mls}$ of blood were removed. Twin right-sided fimbrial ectopic pregnancy was encountered. The fetuses shared a placenta but were in separate amniotic sacs. Right salpingectomy was done. The uterus was observed to as being enlarged to about 18 weeks and had multiple subserosal and intramural leiomyoma. Both ovaries were normal. The Left fallopian was normal. The patient remained stable after the operation and was discharged on the third post-operative day. The specimen obtained was taken for analysis in the pathology laboratory where monochorionic monozygotic twin tubal pregnancy was confirmed.

\section{DISCUSSION}

UTTP is one of the uncommon types of ectopic pregnancies (Chukus et al., 2015). Other unusual types include ectopic pregnancies in the cervix, interstitial segment of the fallopian tube, scar from a prior cesarean delivery, uterine myometrium, ovary, and peritoneal cavity, bilateral ectopic pregnancy as well as heterotopic ectopic pregnancies. In the latter, there is an intrauterine pregnancy co-existing with an ectopic one.

In 1923, Arey and IIl, suggested that tubal ectopic pregnancies occur as a result of "inflammatory changes" on the tube, which precede lodgment of the ovum. These changes consequently delay the ovum in its progress. Risk factors for tubal ectopic include pelvic inflammatory disease especially chlamydia trachomatis infection, smoking, previous tubal surgery, induced conception cycle, congenital anomalies and endometriosis (Rana et al., 2013). These risk factors are thought to cause inflammatory changes on the tube and subsequent scarring and distortion.

The mammalian oviduct is made up of heterogeneous cell types such as ciliated and secretory epithelial cells, and smooth muscle cells. Studies have shown that ciliary beating and smooth muscle activity are the most common factors responsible for moving the embryo through the oviduct (Jansen 1984). The interaction of the embryo and oviduct epithelial cells determines whether there would be a successful transfer into the uterine cavity or a resultant abnormal "homing" of the embryo within the oviduct (Jansen 1984). In addition, a delay of ovum transport and implantation is associated with an increased risk for monozygotic twinning (Bressers et al., 1987). When UTTP or bilateral twin ectopic occur following in vitro fertilization, the timing of the embryo transfer matters such that blastocyst transfer has a higher ectopic implantation rate when compared to cleavage stage transfer (Schwarzler et al., 2004). An embryo can return back into the uterine cavity for implantation but a diseased tube would be prevent this (Herman et al., 1990)

We present a case of UTTP that was not initially detected on ultrasound. The key to diagnosis of an ectopic pregnancy is determining the presence or absence of an intrauterine gestational sac with co-relation of serum B-hCG levels. An ectopic pregnancy should be suspected when TVUS does not show an intrauterine gestation with a serum B-hCG level of $1500 \mathrm{IU} / \mathrm{L}$ or higher. Women with ectopic pregnancies tend to have lower B-hCG levels than those with normal intrauterine pregnancies. In the case of twin ectopic pregnancies however, a high level of B-hCG similar to that seen in normal intrauterine pregnancies occurs, making this correlation difficult (Vohra et al., 2014). TVUS (Trans vaginal ultrasound) is preferred than trans abdominal ultrasound since it allows better visualization of an ectopic mass with or without an embryo within it and detailed evaluation of the adnexa of patients suspected of having ectopic pregnancies. 
The management of UTTP is not different from the guidelines provided for singleton ectopic pregnancies. Surgical and medical management options can be employed guided by the clinical characteristics of the patient. Arikan et al., (2011), presented a case of UTTP treated by a single dose of Methotrexate. These authors recommended that Methotrexate therapy could be offered in tubal twin ectopic pregnancies when the vital signs of the patient are stable and the fetal cardiac activities are negative.
In conclusion, when the diagnosis of an ectopic gestation is made, the possibility of another ectopic pregnancy, either in the same loci or heterotopic, should be considered and management instituted as appropriate. Although UTTP is rare, systematic evaluation, with a high index of suspicion, of the adnexa during ultrasound scanning can be done to avoid missing a second gestational sac. Embryologists and anatomists ought to catalog these uncommon types of ectopic pregnancies in order to sensitize clinicians about their existence.

\section{REFERENCES}

1. Arikan DC, Kiran G, Coskun A, Kostu B. 2011. Unilateral tubal twin ectopic pregnancy treated with single-dose methotrexate. Arch Gynecol Obstet. 283 (2): 397-9

2. Bressers WM, Eriksson AW, Kostense PJ, Parisi P. 1987. Increasing trend in the monozygotic twinning rate. Acta Genet Med Gemellol (Roma). 36 (3):397-408.

3. Chukus A, Tirada N, Restrepo R, Reddy NI. 2015. Uncommon Implantation Sites of Ectopic Pregnancy: Thinking beyond the Complex Adnexal Mass. Radiographics. 35 (3):946-59.

4. De Ott D. 1891. A case of unilateral tubal twin gestation. Annales de Gynecologie et d'Obstetrique. 36: 304.

5. Herman A, Ron-El R, Golan A, Weinraub Z, Bukovsky I, Caspi E. 1990. The role of tubal pathology and other parameters in ectopic pregnancies occurring in in vitro fertilization and embryo transfer. Fertil Steril. 54: 864-8.

6. Jansen RP. 1984. Endocrine response in the fallopian tube. Endocr Rev. 5:525-51.

7. Lema VM. 1990. Unilateral tubal twin pregnancy: a case report East Afr Med J. 67 (12):918-21.

8. Lozeau AM, Potter B. 2005. Diagnosis and management of ectopic pregnancy. Am Fam Physician. 72:1707-14.

9. Panti A, Ikechukwu NE, lukman OO, Yakubu A, Egondu SC, Tanko BA. 2012. Ectopic pregnancy at Usmanu Danfodiyo University Teaching Hospital Sokoto: a ten-year review. Ann Niger Med. 6 (2):87-91.

10. Parker J, Hewson AD, Calder-Mason T, Lai J. 1999. Transvaginal ultrasound diagnosis of a live twin tubal ectopic pregnancy. Australas Radiol. 43:95-7.

11. Rana P, Kazini I, Singh R, Afzal M, Al-Abbasi FA, Aseeri A, Singh R. 2013. Ectopic Pregnancy: A Review. Archives of Gynecology and Obstetrics. 288(4):747-757

12. Schwarzler $P$, Zech $H$, Auer $M$, et al. 2004. Pregnancy outcome after blastocyst transfer as compared to early cleavage stage embryo transfer. Hum Reprod.19:2097-102

13. Vohra S, Mahsood S, Shelton H, Zaedi K, Economides DL. 2014. Spontaneous live unilateral twin ectopic pregnancy - A case presentation. Ultrasound. 22(4): 243-246 